\title{
Interaction of $\beta 1$-adrenoceptor with RAGE mediates cardiomyopathy via CaMKII signaling
}

\author{
Weizhong Zhu, ${ }^{1}$ Sharon Tsang, ${ }^{2}$ David M. Browe, ${ }^{2}$ Anthony Y.H. Woo, ${ }^{3,4}$ Ying Huang, ${ }^{3}$ Chanjuan Xu, ${ }^{5}$ \\ Jian-Feng Liu, ${ }^{5}$ Fengxiang Lv, ${ }^{3}$ Yan Zhang, ${ }^{3}$ and Rui-ping Xiao ${ }^{3,6,7}$ \\ ${ }^{1}$ Nantong University School of Pharmacy, Nantong, China. 'Laboratory of Cardiovascular Science, National Institute on \\ Aging, NIH, Baltimore, Maryland, USA. ${ }^{3}$ State Key Laboratory of Membrane Biology, Institute of Molecular Medicine, \\ Peking University, Beijing, China. ${ }^{4}$ School of Life Sciences and Biopharmaceutics, Shenyang Pharmaceutical University, \\ Shenyang, China. ${ }^{5}$ School of Life Science and Technology, Huazhong University of Science and Technology, Wuhan, China. \\ ${ }^{6}$ Peking-Tsinghua Center for Life Sciences and 'Beijing City Key Laboratory of Cardiometabolic Molecular Medicine, \\ Peking University, Beijing, China.
}

Stimulation of $\beta 1$-adrenergic receptor ( $\beta 1 A R)$, a GPCR, and the receptor for advanced glycation end-products (RAGE), a pattern recognition receptor (PRR), have been independently implicated in the pathogenesis of cardiomyopathy caused by various etiologies, including myocardial infarction, ischemia/reperfusion injury, and metabolic stress. Here, we show that the two distinctly different receptors, $\beta 1 A R$ and RAGE, are mutually dependent in mediating myocardial injury and the sequelae of cardiomyopathy. Deficiency or inhibition of RAGE blocks $\beta 1 A R$ - and RAGE-mediated myocardial cell death and maladaptive remodeling. Ablation or blockade of $\beta 1 A R$ fully abolishes RAGE-induced detrimental effects. Mechanistically, RAGE and $\beta 1 A R$ form a complex, which in turn activates $\mathrm{Ca}^{2+} /$ calmodulin-dependent kinase II (CaMKII), resulting in loss of cardiomyocytes and myocardial remodeling. These results indicate that RAGE and $\beta 1 A R$ not only physically crosstalk at the receptor level, but also functionally converge at the common mediator, CaMKII, highlighting a combined inhibition of RAGE and $\beta 1 A R$ as a more effective therapy to treat diverse cardiovascular diseases, such as myocardial infarction, ischemia/reperfusion injury, and diabetic cardiovascular complications.

Authorship note: Weizhong Zhu, Sharon Tsang, and David M. Browe contributed equally to this work.

Conflict of interest: The authors have declared that no conflict of interest exists.

Submitted: December 6, 2015 Accepted: December 16, 2015 Published: January 21, 2016

Reference information: JCl Insight. 2016;1(1):e84969. doi:10.1172/jci.insight.84969.

\section{Introduction}

The adrenergic system comprising the sympathetic nervous system-catecholamine- $\beta$-adrenergic receptor (SNS-catecholamine- $\beta A R$ ) axis is broadly involved in stress response, energy homeostasis, and muscular actions. The SNS-catecholamine-BAR system is activated in response to physical or emotional stress such as "fight-or-flight" physiological response, which increases cardiac output several-fold within seconds. Under pathological conditions, ischemic or metabolic stress caused by a variety of etiologies also leads to sympathetic overdrive. The increased sympathetic stimulation results in increased cytoplasmic $\mathrm{Ca}^{2+}$ levels, initially producing positive inotropic and chronotropic effects, via the activation of the $\beta 1 \mathrm{AR}-\mathrm{Gs}-\mathrm{adenylyl}$ cyclase-cAMP-protein kinase A signaling cascade. However, persistent $\beta 1 \mathrm{AR}$ stimulation triggers loss of cardiomyocytes and maladaptive remodeling, including myocardial hypertrophy and fibrosis, via mechanisms that depend on $\mathrm{Ca}^{2+}$ /calmodulin-dependent kinase II (CaMKII), independently of protein kinase A $(1,2)$. For instance, myocardial infarction (MI) induced by ligation of the coronary artery leads to sympathetic hyperactivity, which, in turn, triggers myocardial injury and remodeling (3-5). These studies provide a rational basis for the clinical use of $\beta$-blockers in pathological cardiomyopathy, including ischemic cardiomyopathy and diabetic cardiomyopathy (6).

CaMKII, a multifunctional serine/threonine protein kinase (7), is activated canonically by complexing with $\mathrm{Ca}^{2+}$-calmodulin and noncanonically through posttranslational modifications, including phosphorylation, oxidation, and glycosylation in response to neurohormonal, inflammatory, or metabolic stress signals (8-11). Previous studies have shown that transgenic cardiac overexpression of CaMKII- $\delta \mathrm{C}$ is sufficient to induce dilated cardiomyopathy $(12,13)$, whereas transgenic expression of a mitochondria-targeted 

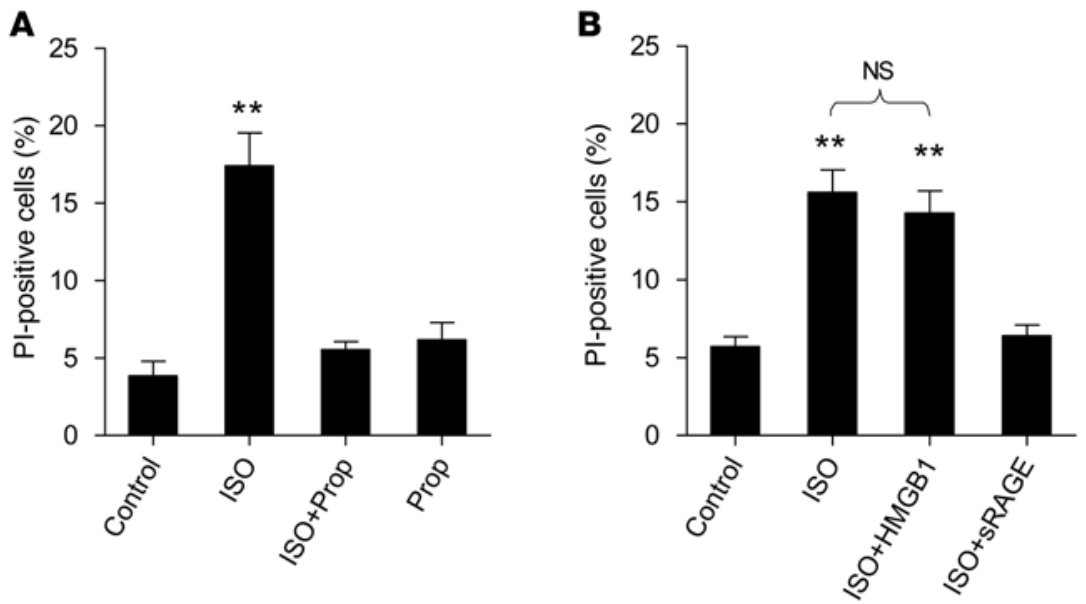

Figure 1. B1AR stimulation elicits cardiomyocyte death via a RAGE-dependent mechanism. (A) ISO-induced increase in PI-positive cells was blocked by the $\beta$ AR blocker Prop. (B) ISO-induced increase in PI-positive cells was abolished by a RAGE decoy, sRAGE. Adult rat cardiomyocytes were subjected to ISO $(1 \mu \mathrm{M})$ plus a $\beta 2 A R$ selective antagonist, ICI $(0.1$ $\mu \mathrm{M})$, in the presence or absence of HMGB1 $(200 \mathrm{ng} / \mathrm{ml})$, Prop $(0.1 \mu \mathrm{M})$, or SRAGE $(14 \mathrm{ng} / \mathrm{ml})$. Cell death was assessed by PI-positive staining. Data were expressed as mean \pm SEM for 4 independent experiments (at least 500 cells from 4 rats for each group); ${ }^{* *} P<0.01$ versus all of the other groups (A) or versus control and ISO + SRAGE (B), 1-way ANOVA. $\beta 1 A R, \beta 1$-adrenergic receptor; RAGE, receptor for advanced glycation end-products; ISO, isoproterenol; PI, propidium iodide; Prop, propranolol; ICI, ICI 118,551; sRAGE, soluble RAGE; HMGB1, high-mobility group box 1 protein.

CaMKII inhibitor protects the heart against ischemia/reperfusion (I/R) injury (14), maladaptive postinfarct remodeling, and proinflammatory signaling (15). Moreover, activation of CaMKII is required for cardiomyocyte necrosis, as well as apoptosis induced by various ischemic and oxidative stress signals $(1,2)$. In particular, we and others have previously shown that excessive $\beta 1 \mathrm{AR}$ stimulation leads to cardiomyocyte death, maladaptive myocardial remodeling, and cardiomyopathy via activation of CaMKII $(1,16)$. Thus, CaMKII constitutes an important pathogenic factor and a potentially important therapeutic target for cardiomyopathy induced by multiple causes.

In addition to the $\beta 1 A R-C a M K I I$ signaling cascade, recent studies have placed the receptor for advanced glycation end-products (RAGE) at the center of pathways for ischemia- and I/R-induced myocardial injury and resultant cardiomyopathy (17). RAGE is a pattern recognition receptor (PRR) and inducibly expressed in various cell types, including cardiomyocytes $(18,19)$, monocytes $(20)$, vascular endothelial cells (21), and vascular smooth muscle cells (22). RAGE is activated by multiple endogenous ligands, including advanced glycation end-products (AGEs) and high-mobility group box 1 protein (HMGB1). Both HMGB1 and AGEs can be passively leaked from necrotic or injured cells as endogenous "danger signals" or actively secreted by cells in response to ischemic, metabolic, or oxidative stress $(15,23,24)$. Interaction of RAGE with these ligands leads to cellular innate immune responses, release of proinflammatory cytokines, generation of ROS, and upregulation of RAGE itself, resulting in a positive feed-forward cycle.

In the heart, RAGE signaling is markedly exaggerated in response to ischemic injury (17), as is the case in the liver and the brain $(17,25,26)$. In particular, MI and I/R injury induce hyperglycemia, which predisposes the cardiovascular system to complications including oxidative stress (27), endothelial dysfunction $(28,29)$, and inflammation $(30,31)$, presumably by increasing blood levels of AGEs. As a result, acute hyperglycemia predicates a higher risk of in-hospital mortality in patients with $\mathrm{MI}$ in the presence or absence of diabetes (32). In contrast, tight glycemic control protects the heart against surgical injuryinduced myocardial inflammation (33).

Previous studies have shown that RAGE signaling can be inhibited by an endogenous decoy, a soluble RAGE (sRAGE). Compared with full-length RAGE, sRAGE is a short variant form of RAGE lacking the transmembrane and cytoplasmic portions. sRAGE inhibits RAGE activation by competitively quenching its ligands $(34,35)$. Similar to the effect of gene ablation of RAGE, sRAGE markedly attenuates MIinduced cardiac inflammation, myocardial damage, and dysfunction (36). The protective effect of sRAGE further indicates that the RAGE-ligand axis plays an essential role in MI- and I/R-induced myocardial injury and cardiomyopathy. 
A

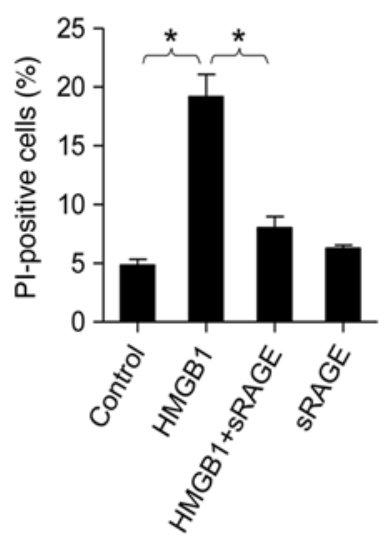

B

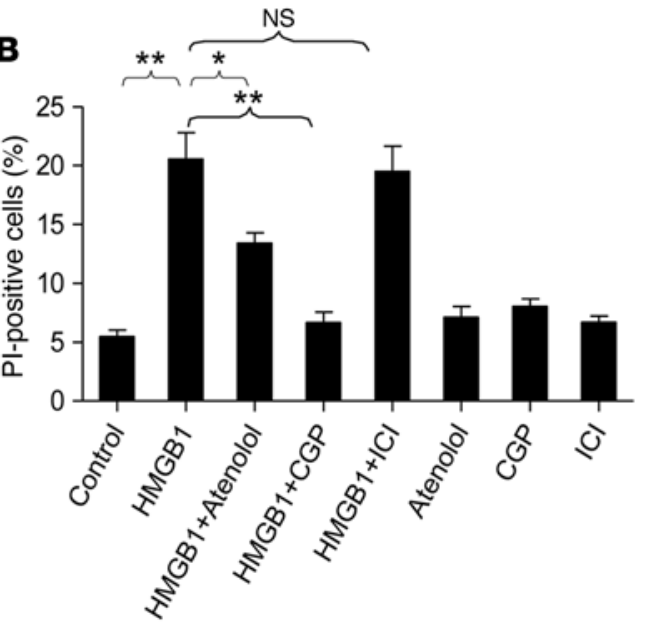

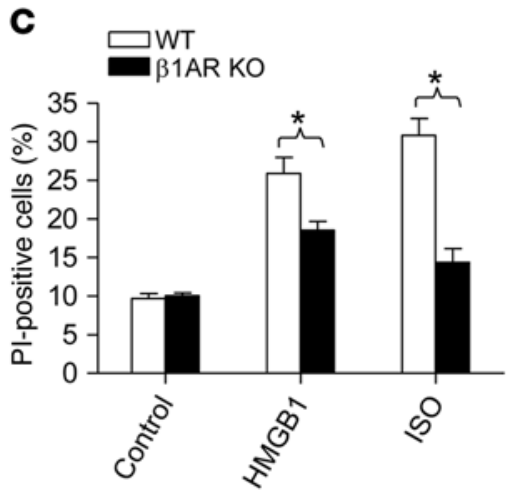

Figure 2. RAGE stimulation with HMGB1 triggers cardiomyocyte death via a $\boldsymbol{\beta 1 A R}$-dependent mechanism. (A) sRAGE abolished HMGB1-induced death of cultured adult rat cardiomyocytes. (B) HMCB1-induced myocyte death was abrogated by $\beta 1 A R$ blockade with atenolol (0.3 $\mu \mathrm{M}$ ) or CGP (0.3 $\mu \mathrm{M}$ ), but not a $\beta 2 A R$ blocker ICI $(0.1 \mu \mathrm{M})$. In both panels, cultured adult rat cardiomyocytes were treated with HMCB1 (200 ng/mI) for 24 hours in the presence or absence of sRAGE or $\beta A R$ blockers. (C) $\beta 1 A R$ deficiency attenuated HMGB1- and ISO-induced death of adult mouse cardiomyocytes. Adult mouse cardiomyocytes were isolated from WT or $\beta 1 A R$ KO mice. Cultured adult mouse cardiomyocytes were treated with HMCB1 (200 ng/ml) or ISO (1 $\mu$ M) for 24 hours. Cell death was assayed by PI staining. Data were expressed as mean \pm SEM for 4 independent experiments (at least 500 cells from 4 rats for each group). ${ }^{*} P<0.05$; ${ }^{*} P<0.01$; 1-way ANOVA. RACE, receptor for advanced glycation end-products; HMCB1, high-mobility group box 1 protein; $\beta 1 A R$, $\beta 1$-adrenergic receptor; sRAGE, soluble RAGE; CGP, CGP 20712A; ICI, ICI 118,551; ISO, isoproterenol; $\beta 1 A R$ KO, $\beta 1$-adrenergic receptor KO; PI, propidium iodide.

Although RAGE and $\beta 1 A R$ have been independently implicated in the pathogenesis of ischemia- and I/R-induced myocardial injury and cardiomyopathy, a relationship between the two receptors is yet to be established. In the present study, we have demonstrated a mutual dependence between $\beta 1 \mathrm{AR}$ and RAGE in mediating cardiomyopathy. Mechanistically, $\beta 1 \mathrm{AR}$ and RAGE form a protein complex, which, in turn, triggers cardiomyocyte death, myocardial remodeling, and heart failure via activating CaMKII.

\section{Results}

$\beta 1 A R$-elicited cardiomyocyte death is RAGE dependent. It is well established that $\beta 1 \mathrm{AR}$ stimulation triggers cardiomyocyte death. In cultured adult rat cardiomyocytes, activation of $\beta 1 \mathrm{AR}$ with isoproterenol (ISO, $1 \mu \mathrm{M}$ ) in the presence of a $\beta 2 \mathrm{AR}$ selective antagonist ICI 118,551 (ICI, $0.1 \mu \mathrm{M}$ ) indeed induced robust cell death, as indexed by the increase of propidium iodide (PI)-positive cells. As expected, this effect was abolished by a $\beta$-blocker, propranolol (Prop, $0.1 \mu \mathrm{M}$ ) (Figure 1A). Importantly, sRAGE, a decoy of RAGE, also effectively abrogated ISO-induced cell death, although stimulation of RAGE with HMGB1 (200 ng/ $\mathrm{ml}$ ) could not further augment ISO-induced cell death (Figure 1B). This result suggests that $\beta 1 \mathrm{AR}$-induced cardiomyocyte death is likely dependent on the activation of RAGE signaling.

Activation of $\beta 1 A R$ is dependent on RAGE-induced cardiomyocyte death. HMGB1 serves as an agonist for RAGE, as well as the TLR. Here we found that HMGB1 (200 ng/ml) markedly increased cardiomyocyte death, which was fully abolished by sRAGE $(14 \mathrm{ng} / \mathrm{ml})$ (Figure 2A), indicating that, under the present experimental conditions, HMGB1 triggers cardiac cell death by activating RAGE. Notably, the effect of HMGB1 on cell death was blocked by $\beta 1 \mathrm{AR}$ selective antagonists, CGP20712A (CGP, $0.1 \mu \mathrm{M})$ and atenolol $(0.1 \mu \mathrm{M})$, but not by a $\beta 2 \mathrm{AR}$ selective antagonist, ICI $(0.3 \mu \mathrm{M})$, (Figure $2 \mathrm{~B})$. Furthermore, using $\beta 1 \mathrm{AR}$-deficient mice and their WT littermates, we corroborated that $\beta 1 \mathrm{AR}$ ablation not only abolished ISO-, but also HMGB1-induced cardiac muscle cell death (Figure 2C).

CaMKII is essential for both $\beta A R$ - and RAGE-mediated cell death signaling. We and others have previously demonstrated that CaMKII is essentially involved in $\beta 1 \mathrm{AR}$-induced death in adult mouse cardiomyocytes in vivo and in culture $(1,2)$. Inhibition of CaMKII activity with a synthetic blocker, KN93 $(0.5 \mu \mathrm{M})$, or a peptide antagonist, AIP $(5 \mu \mathrm{M})$, markedly attenuated ISO-induced cell death in cultured adult rat cardiomyocytes (Figure 3, A and B). It is noteworthy that these CaMKII inhibitors also fully abrogated HMGB1induced myocyte death (Figure 3, C and D), suggesting that $\beta 1 \mathrm{AR}$ - and RAGE-mediated cell death signaling pathways are converged on the common mediator, CaMKII. 
A

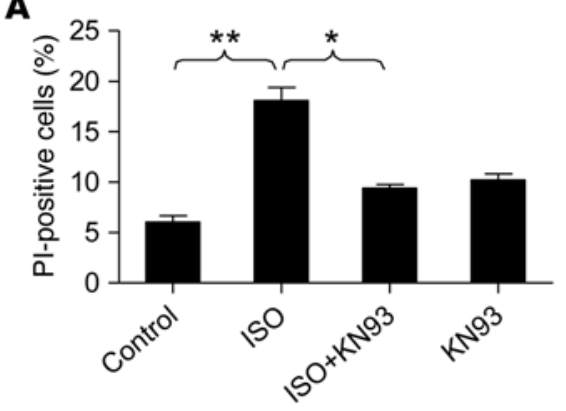

\section{C}

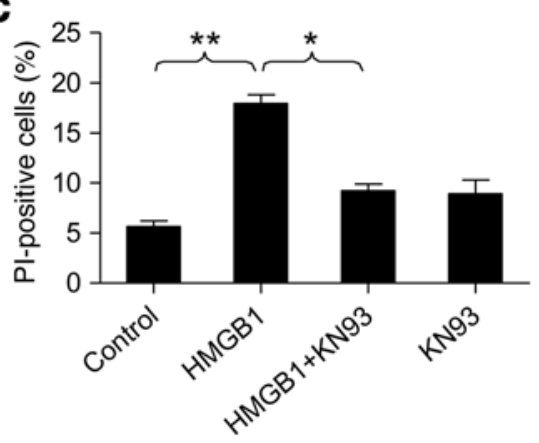

B

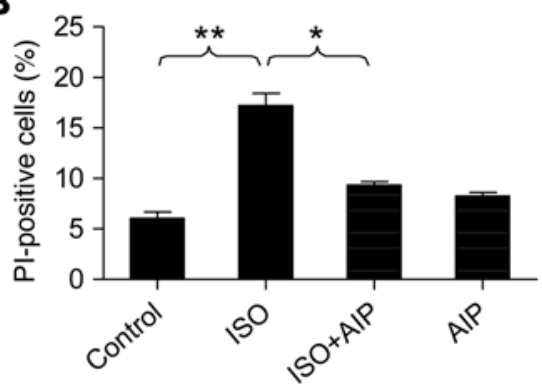

D

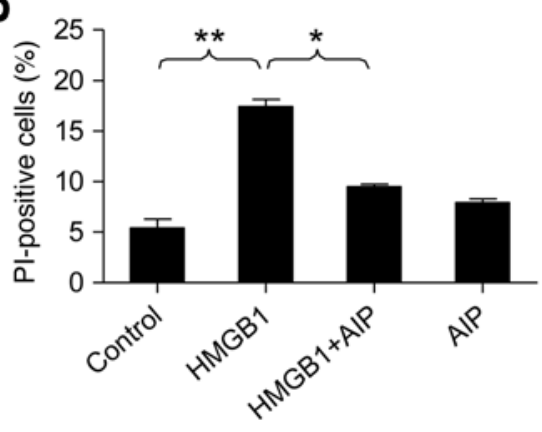

Figure 3. CaMKII is essentially involved in both $\mathbf{B A R}$ - and RAGE-evoked cell death signaling. (A and B) Inhibition of CaMKII with KN93 (A) or AIP (B) blocked ISO-induced death of adult rat cardiomyocytes. (C and D) HMCB1-induced death of cardiomyocytes was also abrogated by the CaMKII inhibitors KN93 (C) and AIP (D). Cultured myocytes were pretreated with either KN93 $(0.5 \mu \mathrm{M})$ or AIP $(5 \mu \mathrm{M})$ for 1 hour and then incubated with ISO $(1 \mu \mathrm{M})$ or HMCB1 $(100 \mathrm{ng} / \mathrm{ml})$ for 24 hours. Data were expressed as mean \pm SEM for 4 independent experiments (at least 500 cells from 4 rats for each group). ${ }^{*} P<0.05$; ${ }^{* *} P<0.01 ; 1$-way ANOVA. CaMKII, Ca+/calmodulin-dependent kinase II; $\beta A R, \beta$-adrenergic receptor; RAGE, receptor for advanced glycation end-products; ISO, isoproterenol; HMGB1, high-mobility group box 1 protein.

RAGE-mediated CaMKII activation is $\beta 1 A R$ dependent. Phospholamban (PLN), a $\mathrm{Ca}^{2+}$ handling protein at sarcoplasmic reticulum, is a well-established endogenous target of CaMKII that phosphorylates PLN at threonine 17 (Thr17). Using the phosphorylation level of Thr17-PLN, P-Thr17-PLN, as an index of CaMKII activity, we found that stimulation of RAGE with HMGB1 $(200 \mathrm{ng} / \mathrm{ml})$ elevated CaMKII activation in a time-dependent manner in cultured rat adult cardiomyocytes (Figure 4A). Pretreatment of cells with sRAGE $(14 \mathrm{ng} / \mathrm{ml})$ abolished HMGB1-induced activation of CaMKII. Importantly, the $\beta 1 \mathrm{AR}$ blocker CGP $(0.1 \mu \mathrm{M})$, also fully abrogated HMGB1-evoked activation of CaMKII (Figure 4B). These results strongly suggest that RAGE-mediated CaMKII activation is $\beta 1$ AR dependent.

Inhibition of RAGE blocks $\beta 1 A R$-induced myocardial cell death and remodeling. To determine the potential pathological relevance of the mutual dependence of RAGE and $\beta 1 A R$ in inducing cardiac cell death, we examined effects of in vivo inhibition of RAGE or $\beta 1 \mathrm{AR}$ using gene-targeted KO mouse models. ISO treatment (i.p., $30 \mathrm{mg} / \mathrm{kg}$ ) markedly elevated blood troponin I level, an index of myocardial injury and myocyte necrosis, in WT mice (Figure 5A). Remarkably, ISO-induced increase in serum troponin I was not only reduced by deficiency of $\beta 1 \mathrm{AR}$ ( $\beta 1 \mathrm{AR}$ KO) but also by ablation of RAGE (RAGE KO) (Figure $5 \mathrm{~A}$ ), highlighting the pathological relevance of the mutual dependence of $\beta 1 \mathrm{AR}$ and RAGE in mediating cardiac cell death and myocardial injury.

To further translate our single cell data into whole animals, we examined cardiac structure and function in response to chronic ISO infusion ( $60 \mathrm{mg} / \mathrm{kg} / \mathrm{d}$ with an osmotic minipump) in the presence or absence of RAGE blockage with sRAGE ( $8 \mathrm{ng} / \mathrm{kg}$, i.p.) for 7 days (Figure 5B). ISO-induced cell death, assessed by the percentage of TUNEL-positive cells, was reduced by cotreatment of the mice with sRAGE (Figure 5, $\mathrm{C}$ and $\mathrm{D}$ ). In addition, ISO infusion for 1 week led to profound myocardial fibrosis (Figure 6, A and B) and reduced cardiac contractility, as manifested by reduced ejection fraction and fractional shortening (Figure 6, C and D). sRAGE markedly ameliorated ISO-induced cardiac fibrosis and restored cardiac contractility (Figure 6). Thus, $\beta 1 \mathrm{AR}$-induced myocardial cell death in vivo, remodeling, and heart failure are also dependent on the activation of RAGE signaling.

Physical association of $\beta 1 A R$ and RAGE. Coimmunoprecipitation assay revealed that $\beta 1 \mathrm{AR}$ and RAGE 

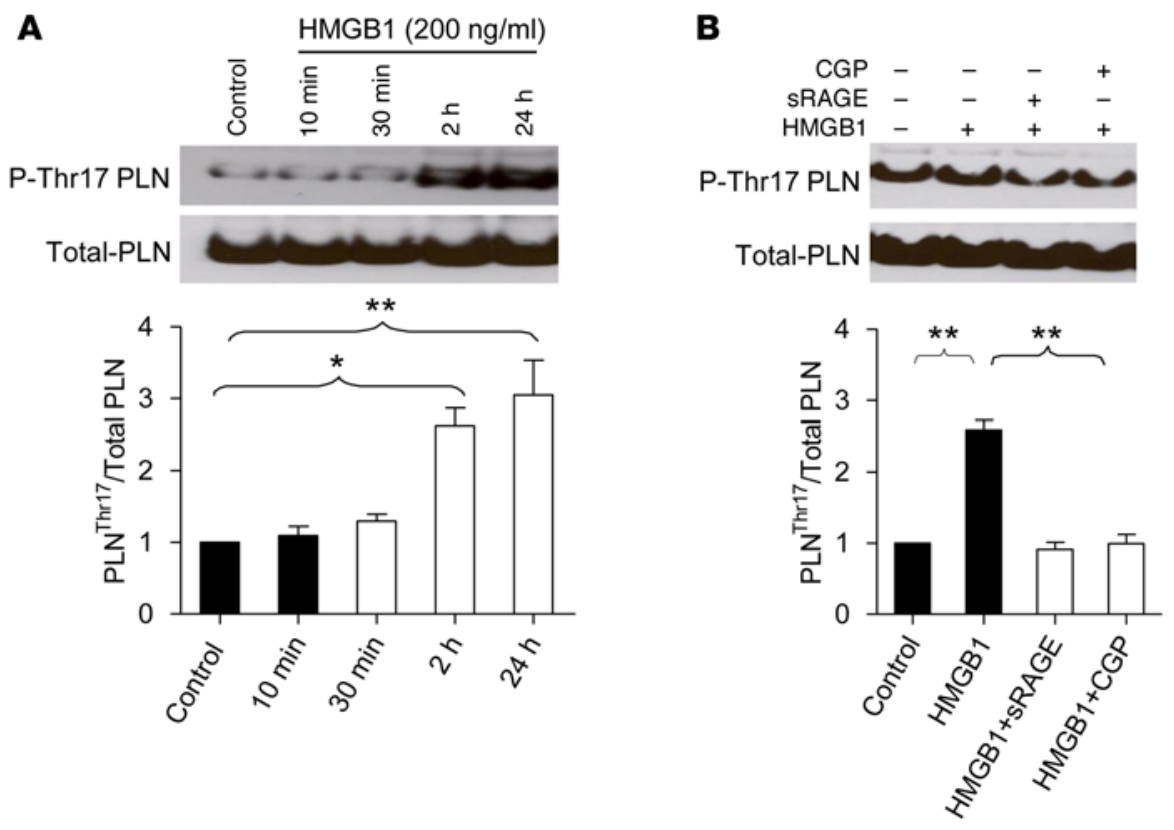

Figure 4. RAGE stimulation with HMGB1 activates CaMKII in a ß1AR-dependent manner. (A) Time course of HMGB1-induced increase in CaMKII activity, as manifested by phosphorylation of its key target, PLN, at Thr17 (P-Thr17 PLN). Top and bottom show the representative and averaged data of Western blots of P-Thr17 PLN and total PLN. Cultured adult rat cardiomyocytes were incubated with HMCB1 (200 ng/ml) for different periods of time. Data were expressed as mean \pm SEM from 3 experiments; ${ }^{*} P<0.05$; ${ }^{* *} P<0.01$; 1 -way ANOVA. (B) HMCB1-induced phosphorylation of the CaMKII target PLN was abolished by sRAGE and $\beta 1 A R$ blockade with CGP, as well. Cells were pretreated with sRAGE $(14 \mathrm{ng} / \mathrm{ml})$ or CGP $(0.3 \mu \mathrm{M})$ for 1 hour and then incubated with HMGB1 $(200 \mathrm{ng} / \mathrm{ml})$ for another 24 hours. Top and bottom show the representative and averaged data of Western blots of P-Thr17 PLN and total PLN, respectively. Data were expressed as mean \pm SEM from 3 experiments. ${ }^{* *} P<0.01 ; 1$-way ANOVA. RAGE, receptor for advanced glycation end-products; HMCB1, high-mobility group box 1 protein; CaMKII, Ca²+calmodulin-dependent kinase II; sRAGE, soluble RAGE; PLN, phospholamban; CGP, CGP 20712A; Thr17, threonine 17; $\beta 1 A R, \beta 1$-adrenergic receptor.

physically interacted with each other in HEK293 cells expressing HA-tagged $\beta 1$ AR and Flag-tagged RAGE, suggesting $\beta 1 \mathrm{AR}$ and RAGE may form a protein complex in intact cells (Figure 7A). Indeed, time-resolved fluorescence resonance energy transfer (TR-FRET) assay showed that coexpression of either HA-SNAP-

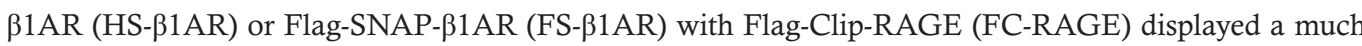
greater FRET intensity compared with that in cells expressing either $\beta 1 A R$ or RAGE alone (Figure 7B), substantiating a physical interaction between the structurally distinct receptors. The enhanced FRET intensity was not caused by the levels of receptor density (Figure 7C). The present in vivo and in vitro data indicate that $\beta 1 \mathrm{AR}$ and RAGE functionally and physically interact with each other, resulting in their mutual dependence in mediating myocardial cell death and the sequelae of cardiomyopathy.

\section{Discussion}

In the present study, there are three major discoveries. First, we have shown that RAGE-induced cardiac cell death is abolished by $\beta 1 \mathrm{AR}$ deficiency or blockade; $\beta 1 \mathrm{AR}$-induced myocardial cell death and remodeling are abrogated by RAGE ablation or the endogenous RAGE decoy, sRAGE. Second, both $\beta 1$ AR- and RAGE-mediated cardiac detrimental effects depend on the activation of CaMKII, an important causal factor of cardiomyopathy and heart failure. Third, $\beta 1 \mathrm{AR}$ and RAGE physically interact with each other, forming a protein complex. These findings indicate that the two distinctly different receptors, $\beta 1 \mathrm{AR}$ (a prototypical member of GPCR family) and RAGE (a member of the PPR family), are mutually dependent in mediating myocardial cell death and cardiomyopathy, and perhaps other chronic stress-related diseases such as diabetes and cancer, as well.

It has been previously reported that membrane receptors can undergo homodimerization or heterodimerization, resulting in a highly diversified as well as integrated response to pathological perturbations or physiological signals. A close inspection reveals that the dimerization often occurs within the same class or closely related classes of receptors. For instance, we and others have previously reported that $\beta 2 \mathrm{AR}$ may exist as homodimers (37) or $\beta 1 \mathrm{AR}-\beta 2 \mathrm{AR}$ heterodimers (38) to enhance signaling diversity and accuracy. 
A

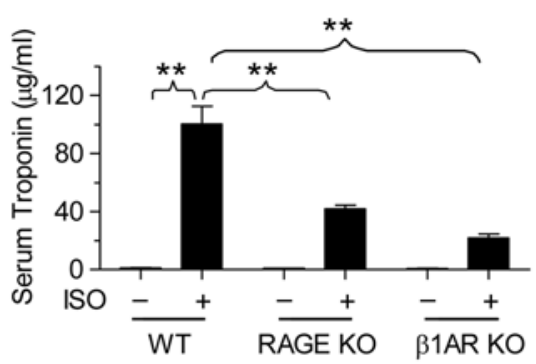

B

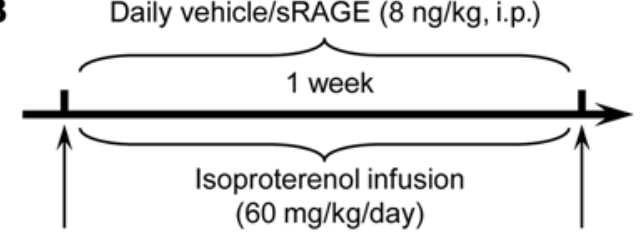

Osmotic pump implantation
Echo and sacrifice for tissue collection
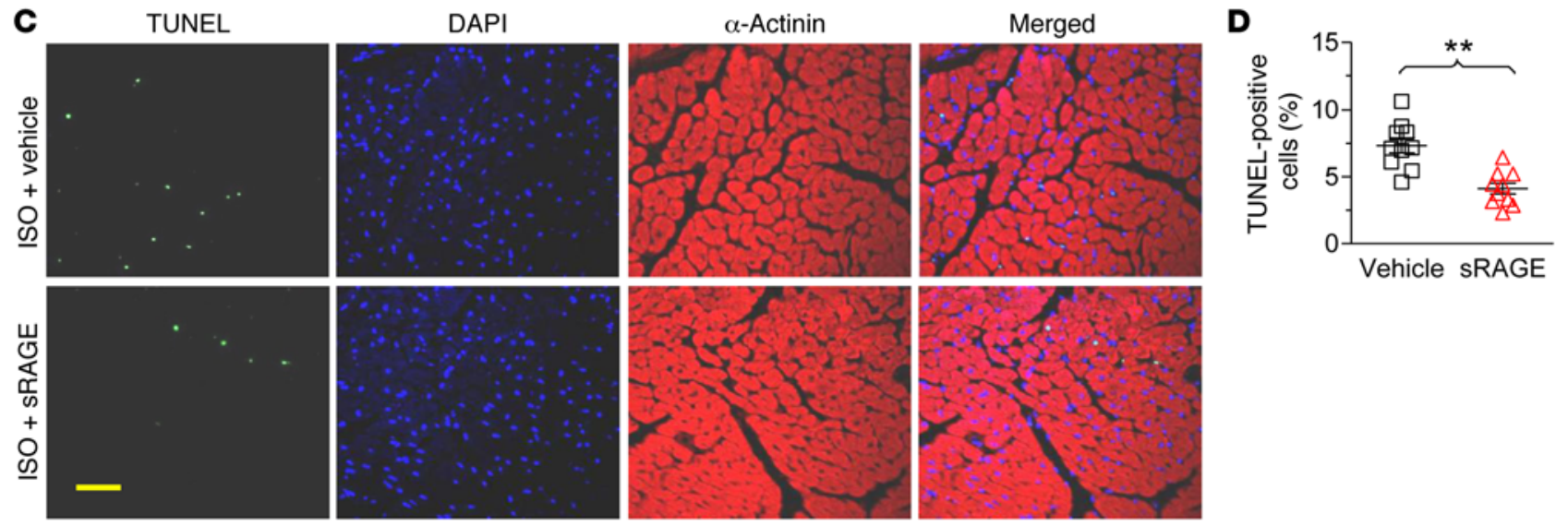

Figure 5. Inhibition of RAGE signaling with sRAGE or RAGE ablation blocks ISO-induced necrotic and apoptotic cell death. (A) Deletion of RAGE diminished acute ISO treatment induced myocardial necrotic cell death, indexed by increased serum troponin I levels. Mouse serum was collected after 24-hour treatment of ISO (30 mg/kg, i.p.). Serum tropinin I was measured by ELISA (Abace-Biology). The averaged data were expressed as mean \pm SEM from 4 mice for each group. ${ }^{*} P<0.01$ as indicated; 1-way ANOVA. (B) Schematic presentation showing the experimental protocol for chronic ISO infusion in the presence or absence of cotreatment with daily sRAGE injection. (C and $\mathbf{D})$ Treatment of mice with sRAGE prevents chronic ISO treatment-induced myocardial apoptotic cell death assessed by TUNEL-positive staining. Representative TUNEL staining (C) and the averaged data (D). Data were expressed as mean \pm SEM from 10 mice of 3 independent experiments. ${ }^{*} P<0.01$ versus vehicle; Student's $t$ test. Mice were subjected to chronic ISO treatment $(60 \mathrm{mg} / \mathrm{kg} / \mathrm{d}$ by minipump) in the presence or absence of sRAGE ( $8 \mathrm{ng} / \mathrm{kg} / \mathrm{d} \mathrm{i.p.)} \mathrm{for} 7$ days and then sacrificed for myocardial TUNEL staining. Scale bar: $20 \mu \mathrm{m}$. RAGE, receptor for advanced glycation end-products; SRAGE, soluble RAGE; ISO, isoproterenol; $\beta 1 A R$ KO, $\beta 1$-adrenergic receptor KO.

The present study has, however, revealed a new mode of physical interaction between membrane receptors, i.e., dimerization or polymerization of receptors from two distinctly different families (a GPCR and a PRR). Specifically, using coimmuoprecipitation and FRET assays, we have provided physical evidence for the formation of a protein complex of $\beta 1 A R$ and RAGE. Functionally, sRAGE blocks not only RAGE- but also $\beta 1 A R$-induced cardiomyocyte death; $\beta 1 A R$ deficiency or blockade abolishes RAGE- and $\beta A R$-mediated cell death, underscoring the mutual dependence of $\beta 1 \mathrm{AR}$ and RAGE in triggering cardiac cell death and cardiomyopathy. These novel findings shed new light on receptor signaling theory.

Although they belong to different receptor families, both $\beta 1 A R$ and RAGE are stress sensors and activated by neurohormonal and metabolic stress stimuli. During cardiac ischemic or metabolic stress, plasma catecholamines, including norepinephrine and epinephrine, are significantly increased (3-5), which further damages the myocardium. Equally important, cardiac stress is accompanied by acute hyperglycemia, which is closely associated with infarct area expansion, worsened cardiac function, and poor prognosis in both diabetic and nondiabetic patients with MI $(21,22,39)$. Epidemiological studies have established a positive correlation between blood concentrations of RAGE ligands, AGEs and HMGB1, and heart failure (40-43), implying a causal relationship between RAGE signaling and myocardial damage. Indeed, AGEs and RAGE expression level are increased in isolated perfused hearts in response to I/R injury, whereas inhibition of RAGE signaling with sRAGE or gene ablation markedly ameliorates I/R-induced myocardial injury, regardless of the presence or absence of diabetes $(17,44)$. These studies suggest that exaggerated RAGE signaling contributes to the pathogenesis of cardiomyopathy and heart failure. Importantly, our present in vivo and in vitro data indicate that catecholamine-mediated cardiac injury is also RAGE dependent, unraveling a previously unappreciated mechanism underlying the beneficial effect of RAGE 


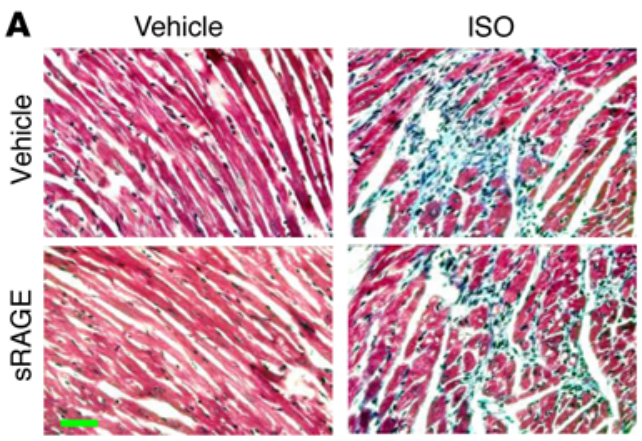

C

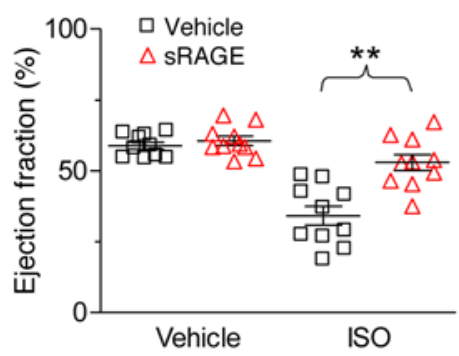

B

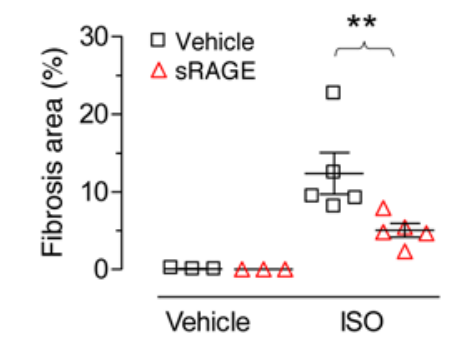

D

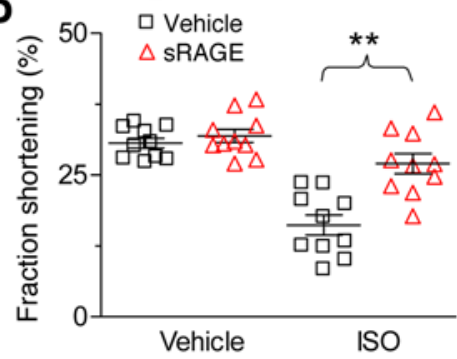

Figure 6. SRAGE inhibits ISO-induced myocardial fibrosis and contractile dysfunction. (A and B) The representative images (A) and averaged data (B) of cardiac fibrosis induced by ISO infusion in the presence or absence of sRAGE treatment. Data were expressed as mean \pm SEM from 10 mice of 3 independent experiments; ${ }^{*} P<0.01$; 1-way ANOVA. Scale bar: $50 \mu \mathrm{m}$. (C and D) SRAGE administration restored ISO-suppressed cardiac contractile function, as manifested by preserved ejection fraction (C) and fractional shortening (D). Mice were treated with ISO (60 $\mathrm{mg} / \mathrm{kg} / \mathrm{d}$ delivered by osmotic minipump) in the presence or absence of SRAGE ( $8 \mathrm{ng} / \mathrm{kg} / \mathrm{d}$, i.p.) for 7 days. Data were expressed as mean \pm SEM from 10 mice of 3 independent experiments; ${ }^{* *} P<0.01$; 1-way ANOVA. SRAGE, soluble receptor for advanced glycation end-products; ISO, isoproterenol.

blockade. Ablation or blockade of $\beta 1 \mathrm{AR}$ fully abolishes RAGE-induced detrimental effects, revealing a novel beneficial effect of $\beta$-blocker therapy in the context of metabolic stress-related diseases.

Clinical studies have shown that the risk of cardiovascular diseases, including coronary heart disease (CHD), cerebrovascular disease, and peripheral vascular disease, are increased by 2 - to 3-fold in patients with diabetes and that $80 \%$ of patients with diabetes die from cardiovascular complications, with CHD as the leading cause of death. $\beta$-Blocker therapy is a mainstay of the current treatment for CHD and MI $(45,46)$, and exhibits a greater beneficial effect in patients with diabetes $(6,47,48)$. In line with the present findings, it has been shown that a long-term $\beta$-blocker therapy decreases serum levels of multiple RAGEregulated inflammatory factors, including IL-6, IL-10, and TNF- $\alpha$, in patients with heart failure (49). These previous observations might be explained by the cross-talk and mutual dependence of RAGE and $\beta 1 A R$ signaling. Since both $\beta 1 A R$ and RAGE signaling pathways are overdriving in stressful cardiac conditions — such as MI and I/R injury - in the presence or absence of diabetes, inhibition of the hyperactivated RAGE signaling is expected to improve the structure and function of the failing or injured heart, similar to $\beta$-blocker therapy. Indeed, inhibition of RAGE with sRAGE exhibits therapeutic effects in multiple disease models with cardiac pathological conditions $(50,51)$. Equally appealing, $\beta$-blocker therapy may be used to treat RAGE-related diseases such as chronic inflammation, diabetes and its cardiovascular complications, and perhaps cancers, as well. This possibility awaits future investigation.

Mechanistically, we have identified CaMKII as a mediator that converges both RAGE- and B1ARevoked inflammatory cell death (necrotic) signaling. Over the past two decades, it has been consistently documented that activation of CaMKII is required for $\beta 1$ AR-evoked myocardial apoptosis $(1,2,16)$, hypertrophy (52), and cardiac remodeling (53). Apart from the receptor-mediated and $\mathrm{Ca}^{2+} / \mathrm{calmod}_{-}$ ulin-dependent activation, CaMKII is activated by posttranslational modulations, including oxidation, glycosylation, and autophosphorylation, in response to ischemic or metabolic stress, contributing to those pathogenic stimuli-induced myocardial injury (9), inflammation (54), and remodeling (11). For instance, diabetic hyperglycemia causes cardiac arrhythmia by enhanced O-linked glycosylation of CaMKII (55), and increases post-MI mortality by elevated CaMKII oxidation $(9,56)$. On top of these previous studies, we have now demonstrated a function of CaMKII in converging RAGE- and $\beta 1 \mathrm{AR}$-induced cardiac cell 
A
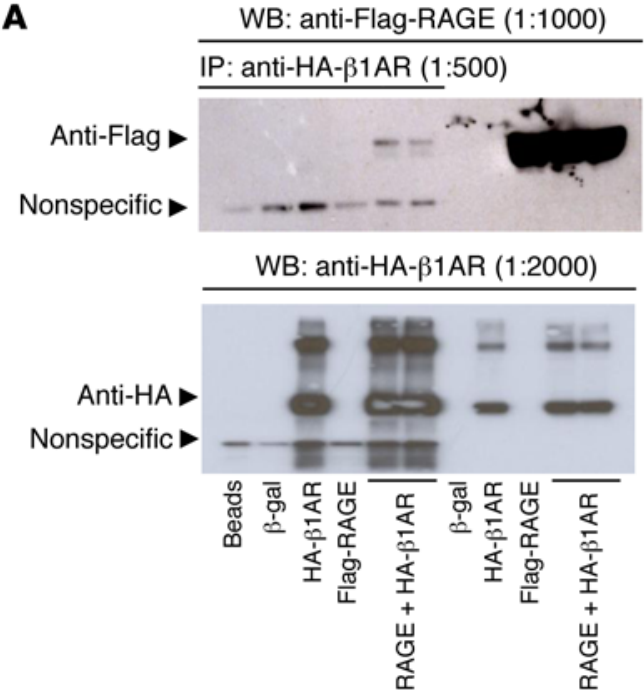

B

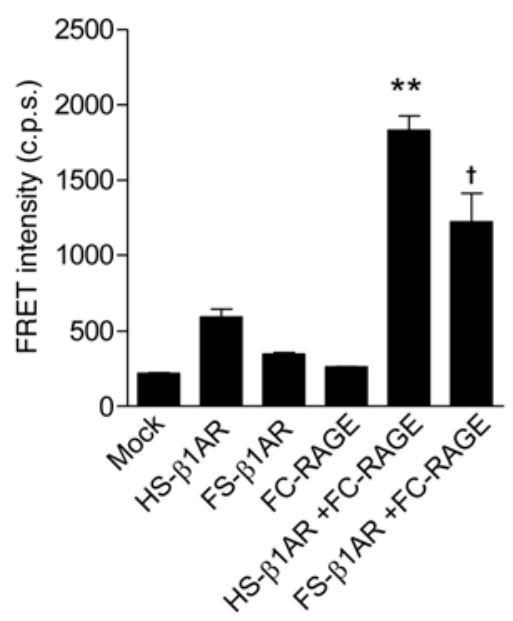

C
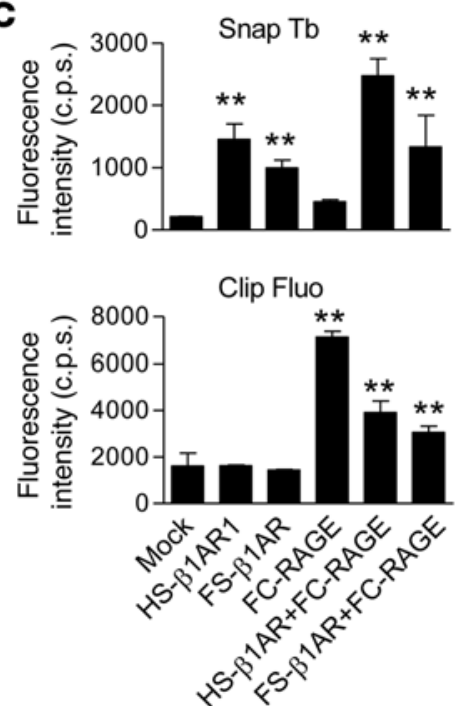

Figure 7. Dimerization of $\beta 1 A R$ and RAGE in HEK293 cells coexpressing $\beta 1 A R$ and RAGE. (A) Coimmunoprecipitation of $\beta 1 A R$ with RAGE to indicate the interaction between these two receptors. HEK293 cells were transfected with either Flag-RACE or HA- $\beta 1 A R$ or both. Forty-eight hours after transfection, total cellular proteins were used for immunoprecipitation assay with anti-HA (for $\beta 1 A R$ ) $(1: 1,000)$ overnight. The precipitation was followed by Western blotting with anti-Flag (for RACE) (1:1,000). The cellular lysate protein $(30 \mu \mathrm{g})$ was used as a positive control for Flag-RAGE expression and anti-HA for loading control. The experiments were repeated 3 times. (B) FRET intensities in HEK2 233 cells' coexpression of either HS- $\beta 1 A R$ or FS- $\beta 1 A R$ with FC-RAGE were expressed as the interaction of proteins between Snap and Clip. ${ }^{\dagger} P<0.05$ versus HS- $\beta 1$ AR or FC-RAGE; ${ }^{* *} P<0.01$ versus FS- $\beta 1$ AR or FC-RACE; 1 -way ANOVA. (C) Cell surface expression of $\beta 1 A R$ and RAGE detected by Snap substrate (top panel) or Clip (bottom panel) fluorescence. ${ }^{* *} P<0.05$ versus Mock; 1 -way ANOVA. The averaged data were expressed as mean \pm SEM from 3 individual experiments with triplicates. $\beta 1 A R$, $\beta 1$-adrenergic receptor; RAGE, receptor for advanced glycation end-products; HEK, human embryonic kidney; FRET, fluorescence resonance energy transfer; HS- $\beta 1 A R$, HA-SNAP- $\beta 1 A R$;

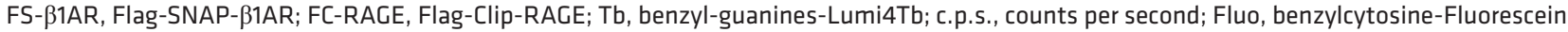

death signaling, providing a key mechanism synergizing sympathetic overdrive- and metabolic stressinduced tissue damage. Nevertheless, future study is required to determine what is the exact mechanism(s) underlying the functional cross-talk and, more specifically, whether and how $\beta 1 \mathrm{AR}$ and RAGE undergo heterodimerization in myocardium.

In summary, we have shown, for the first time to our knowledge, that RAGE and $\beta 1 A R$ are mutually dependent in inducing cardiomyocyte death, maladaptive remodeling, and cardiomyopathy. Mechanistically, RAGE and $\beta 1 \mathrm{AR}$ form a protein complex that activates the common downstream signaling molecule CaMKII, resulting in myocardial cell death and remodeling in response to hormonal or metabolic stress, a situation often evoked by MI, I/R injury, pressure overload, or diabetes. Targeting RAGE as well as $\beta 1 \mathrm{AR}$ has far-reaching pathogenic and therapeutic implications.

\section{Methods}

Animals were maintained in the Center for Experimental Animals (an Association for Assessment and Accreditation of Laboratory Animal Care-accredited experimental animal facility) at Peking University, Beijing, China. The animals were randomly allocated to experimental groups. Only male animals were used. No noninclusion or exclusion parameters were used in our studies. We did not blind investigators to treatments, but no subjective assessments were made.

Preparation of $S R A G E$. The construction of the expression vector for sRAGE (a truncated form of RAGE containing amino acid 23-340) has been described previously (57). The construct was used to establish the stable T7-tagged sRAGE CHO-K1 cell lines (catalog CCL-61, American Type Culture Collection [ATCC]). The culture medium containing sRAGE was collected. A Novagen T7 tag affinity purification kit was used to purify sRAGE, according to the manufacturer's instruction. The concentration of purified sRAGE was determined using a RAGE ELISA kit (R\&D Systems) against an in-house standard standardized in our previous study (57).

Isolation and culture of adult cardiomyocytes. Single cardiomyocytes were isolated from the hearts of 2to 3-month-old male mice (C57BL/6) or rats (Sprague-Dawley). Enzymatic digestion, cell isolation, and culture were performed as described previously (58). Briefly, myocytes were plated at 0.5 to $1 \times 10^{4} / \mathrm{cm}^{2}$ 
with minimal essential medium containing $1.2 \mathrm{mM} \mathrm{Ca}^{2+} / 2.5 \% \mathrm{FBS} / 1 \%$ penicillin-streptomycin in culture dishes precoated with $10 \mu \mathrm{g} / \mathrm{ml}$ mouse laminin. Three hours after culture, the myocytes were treated with a $\beta 1 \mathrm{AR}$ antagonist (Prop, $1 \mu \mathrm{M})$ or sRAGE $(14 \mathrm{ng} / \mathrm{ml})$ for 1 hour before the addition of ISO $(1 \mu \mathrm{M})$ or HMGB1 (100-200 ng/ml). End-point assays were performed 24 hours after ISO or HMGB1 treatment, unless otherwise indicated. All dishes were supplemented with ascorbic acid (100 $\mu \mathrm{M}$, Sigma-Aldrich) to prevent the oxidation of ISO. $\beta 1 \mathrm{AR}$ KO mice were provided by Brian Kobilka at Stanford University (Stanford, California, USA) (38).

PI staining. Cell death was determined by PI staining. The stimulated cardiomyoytes were incubated with $10 \mu \mathrm{g} / \mathrm{ml}$ of PI for 15 minutes and visualized by fluorescence microscopy. The PI-positive and total cells were counted in at least 8 random fields. The extents of cell death were expressed in terms of percentages of PI-positive cells.

Isoproterenol-induced mouse heart failure. Heart failure model was established by s.c. injection of ISO (60 mg/kg/d, dissolved in saline, Sigma-Aldrich) with osmotic pump (ALZET) for 7 consecutive days. The control animals were given vehicle (saline containing $0.5 \mathrm{mM}$ ascorbic acid) during the same period. M-mode echocardiograms were taken 7 days after ISO infusion. For sRAGE treatment, recombinant sRAGE was delivered by daily i.p. injection $(8 \mathrm{ng} / \mathrm{kg} / \mathrm{d})$. The animals were randomly allocated to experimental groups. Only male mice (C57BL/6) at the age of 8-12 weeks were used. RAGE KO mice were provided by Angelika Bierhaus (German Cancer Research Center, Heidelberg, Germany).

Coimmunoprecipation. HEK293 cells (catalog CRL-1573, ATCC) grown to 60\% confluency in 60-mm cell culture dishes were transfected with $1 \mu \mathrm{g}$ of Flag-RAGE plasmid (gift from Lin Li at Laboratory of Cardiovascular Science, National Institute on Aging, NIH, Bethesda, Maryland, USA). After 24 hours, cells were infected with adenovirus containing HA- $\beta 1 \mathrm{AR}$. Another 24 hours later, cells were lysed with IP buffer (150 $\mathrm{mM} \mathrm{NaCl}, 20 \mathrm{mM}$ Tris-Cl, $10 \mathrm{mM}$ EDTA, 1\% NP-40, $1 \mathrm{mM}$ Na3VO4, 1× protease inhibitor cocktail, $\mathrm{pH}$ 7.4). Cell lysates $(200 \mu \mathrm{g})$ in $300 \mu \mathrm{l}$ of reaction buffer were incubated with $0.5-1.0 \mu \mathrm{g}$ of anti-Flag (SigmaAldrich, clone M2) antibody and $30 \mu 1$ of Protein A/G PLUS Agarose beads overnight at $4^{\circ} \mathrm{C}$. The beads were then washed 3 times with IP buffer. After the final wash, the beads were suspended in Laemmli buffer and the eluates were subjected to SDS-PAGE. Cell lysates $(50 \mu \mathrm{g})$ were also loaded in separate lanes as input controls. Proteins were transferred to PVDF membranes by Western blotting and detected with anti-HA (Covance) and anti-Flag antibodies.

TR-FRET assay. HEK293 cells were transfected with plasmids containing SNAP-tagged or Clip-tagged receptors by electroporation and were plated in polyornithine-coated, black-walled, black-bottom, 96-well plates at $10^{5}$ cells per well. Twenty-four hours after transfection, adherent cells were washed with culture medium and incubated with $100 \mu 1$ per well of culture medium containing SNAP substrates (BG-Lumi4Tb, $0.3 \mu \mathrm{M})$ or Clip substrate (1 $\mu \mathrm{M}$ BC-Fluorescein), or SNAP substrates (BG-Lumi4Tb, $0.3 \mu \mathrm{M})$ plus Clip substrate $\left(1 \mu \mathrm{M}\right.$ BC-Fluorescein), for 1 hour at $37^{\circ} \mathrm{C}$. Cells were then washed 3 times with warm Tris-Krebs buffer (20 mM Tris, pH 7.4, $118 \mathrm{mM} \mathrm{NaCl}, 1.2 \mathrm{mM} \mathrm{KH}_{2} \mathrm{PO}_{4}, 1.2 \mathrm{mM} \mathrm{MgSO}_{4}, 4.7 \mathrm{mM} \mathrm{KCl}, 1.8 \mathrm{mM}$ $\mathrm{CaCl}_{2}$ ). After washing, the cells were incubated with Tris-Krebs buffer, and fluorescence and FRET were read using an Infinite F500 spectrofluorometer (Tecan). Fluorescence of Lumi4Tb (excitation at $340 \mathrm{~nm}$, emission at $620 \mathrm{~nm}, 50$ - $\mu$ s delay, 400- $\mu$ s integration time, gain of 100) and BC-Fluorescein (excitation 485 $\mathrm{nm}$, emission $520 \mathrm{~nm}, 0-\mu$ s delay, 1,000- $\mu$ s integration time, gain of 50), and raw TR-FRET values (excitation at $340 \mathrm{~nm}$, emission at $520 \mathrm{~nm}, 50-\mu$ s delay, 400- $\mu$ s integration time, gain of 100) were measured. The experiments were repeated 3 times, and each assay condition was done in triplicate.

Materials. Unless otherwise indicated, all chemicals were purchased from Sigma-Aldrich. Anti-PLN and anti-phosphorylated PLN were purchased from PhosphoProtein Research (A010-14 and A010-13). Anti-HA was from Covance Research Products Inc. (MMS-101P). Anti-Flag was purchased Sigma-Aldrich (F7425). HMGB1 were purchased from R\&D Systems (1690-HMB-050). Troponin I ELISA kit was purchased from Abace-Biology (DEIA6156). Reagents for the TR-FRET assay were manufactured by Cisbio Bioassays.

Statistics. Data are expressed as the mean \pm SEM. Statistical analysis was performed with GraphPad PRISM version 5.01 (GraphPad Software Inc.) and the SPSS 18.0 software package (SPSS Inc.). Data sets were tested for normality of distribution with Kolmogorov-Smirnov test. Data groups (2 groups) with normal distribution were compared using 2-sided unpaired Student's $t$ tests. The Mann-Whitney $U$ test was used for nonparametric data. Comparisons between multiple groups were assessed by 1-way ANOVA with Bonferroni post hoc analysis. The $P$ values are presented in figure legends where a statistically significant difference was found: ${ }^{*} P<0.05 ;{ }^{*} P<0.01$. No statistical method was used to predetermine sample size. 
Study approval. All procedures involving experimental animals were performed in accordance with protocols approved by the Committee for Animal Research of Peking University, Beijing, China, and conformed to the Guide for the Care and Use of Laboratory Animals (Guide for the Care and Use of Laboratory Animals. 8th ed. The National Academies Press. 2011.).

\section{Author contributions}

WZ, ST, and DMB are equally contributing first authors. WZ, ST, DMB, YH, CX, JFL, FL, and YZ conducted key experiments. WZ, AYHW, JFL, YZ, and RPX designed the study. WZ, AYHW, YZ, and RPX analyzed the data and wrote the manuscripts.

\section{Acknowledgments}

This project was supported by the National Basic Research Program of China (2013CB531200 to Y. Zhang, 2012CB518000 to R.P. Xiao, and 2012CB910402 to A.Y.H. Woo), The National Natural Science Foundation of China (81270190 to F. Lv, 81370345 to W. Zhu, 81130073 to R.P. Xiao, 81170100 to Y. Zhang, 81370234 to Y. Zhang, and 31221002 to R.P. Xiao), and National Science and Technology Major Projects for "Major New Drugs Innovation and Development" (2013ZX09508104 to R.P. Xiao, 2013ZX09507001 to Y. Zhang). We thank Edward G. Lakatta and Ling Li at the National Institute on Aging, NIH, USA, for providing reagents and insightful discussions.

Address correspondence to: Rui-Ping Xiao or Yan Zhang, Institute of Molecular Medicine, Peking University, Beijing 100871, China. Phone: 86.10.62757243; E-mail: xiaor@pku.edu.cn (R.P. Xiao). Phone: 86.10.62754557; E-mail: zhangyan9876@pku.edu.cn. (Y. Zhang).

1. Zhu WZ, et al. Linkage of $\beta 1$-adrenergic stimulation to apoptotic heart cell death through protein kinase A-independent activation of $\mathrm{Ca}^{2+} /$ calmodulin kinase II. J Clin Invest. 2003;111(5):617-625.

2. Zhu WZ, et al. Activation of CaMKII $\delta \mathrm{C}$ is a common intermediate of diverse death stimuli-induced heart muscle cell apoptosis. J Biol Chem. 2007;282(14):10833-10839.

3. Du XJ, Cox HS, Dart AM, Esler MD. Sympathetic activation triggers ventricular arrhythmias in rat heart with chronic infarction and failure. Cardiovasc Res. 1999;43(4):919-929.

4. Graham LN, Smith PA, Huggett RJ, Stoker JB, Mackintosh AF, Mary DA. Sympathetic drive in anterior and inferior uncomplicated acute myocardial infarction. Circulation. 2004;109(19):2285-2289.

5. Zhang W, Huang BS, Leenen FH. Brain renin-angiotensin system and sympathetic hyperactivity in rats after myocardial infarction. Am J Physiol. 1999;276(5 pt 2):H1608-H1615.

6. Garcia-Egido A, et al. Beta-blocker therapy and prognosis of heart failure patients with new-onset diabetes mellitus. Int J Clin Pract. 2015;69(5):550-559.

7. Braun AP, Schulman H. The multifunctional calcium/calmodulin-dependent protein kinase: from form to function. Annu Rev Physiol. 1995;57:417-445.

8. De Koninck P, Schulman H. Sensitivity of CaM kinase II to the frequency of $\mathrm{Ca}^{2+}$ oscillations. Science. 1998;279(5348):227-230.

9. Erickson JR, et al. A dynamic pathway for calcium-independent activation of CaMKII by methionine oxidation. Cell. 2008;133(3):462-474.

10. Mangmool S, Shukla AK, Rockman HA. $\beta$-Arrestin-dependent activation of $\mathrm{Ca}^{2+} /$ calmodulin kinase II after $\beta 1$-adrenergic receptor stimulation. J Cell Biol. 2010;189(3):573-587.

11. He BJ, et al. Oxidation of CaMKII determines the cardiotoxic effects of aldosterone. Nat Med. 2011;17(12):1610-1618.

12. Zhang $\mathrm{T}$, et al. The $\delta \mathrm{C}$ isoform of CaMKII is activated in cardiac hypertrophy and induces dilated cardiomyopathy and heart failure. Circ Res. 2003;92(8):912-919.

13. Sossalla S, et al. Diastolic dysfunction and arrhythmias caused by overexpression of CaMKII $\delta(\mathrm{C})$ can be reversed by inhibition of late $\mathrm{Na}^{+}$current. Basic Res Cardiol. 2011;106(2):263-272.

14. Joiner ML, et al. CaMKII determines mitochondrial stress responses in heart. Nature. 2012;491(7423):269-273.

15. Hori O, et al. The receptor for advanced glycation end products (RAGE) is a cellular binding site for amphoterin. J Biol Chem. 1995;270(43):25752-25761.

16. Yang Y, et al. Calmodulin kinase II inhibition protects against myocardial cell apoptosis in vivo. Am J Physiol Heart Circ Physiol. 2006;291(6):H3065-H3075.

17. Bucciarelli LG, et al. Receptor for advanced-glycation end products: key modulator of myocardial ischemic injury. Circulation. 2006;113(9):1226-1234.

18. Vesikansa A, et al. Expression of GluK1c underlies the developmental switch in presynaptic kainate receptor function. Sci Rep. 2012;2:310

19. Schmidt AM, et al. Advanced glycation endproducts interacting with their endothelial receptor induce expression of vascular cell adhesion molecule-1 (VCAM-1) in cultured human endothelial cells and in mice. J Clin Invest. 1995;96(3):1395-1403.

20. Huttunen HJ, Kuja-Panula J, Sorci G, Agneletti AL, Donato R, Rauvala H. Coregulation of neurite outgrowth and cell survival by amphoterin and S100 proteins through receptor for advanced glycation end products (RAGE) activation. J Biol Chem. 
2000;275(51):40096-40105.

21. Lazaros G, et al. Distinct association of admission hyperglycemia with one-year adverse outcome in diabetic and non-diabetic patients with acute ST-elevation myocardial infarction. Hellenic J Cardiol. 2013;54(2):119-125.

22. Usami M, et al. Effect of intracoronary thrombectomy on 30-day mortality in non-diabetic patients with acute hyperglycemia after acute myocardial infarction. J Cardiol. 2009;53(3):429-436.

23. Huttunen HJ, Kuja-Panula J, Rauvala H. Receptor for advanced glycation end products (RAGE) signaling induces CREBdependent chromogranin expression during neuronal differentiation. J Biol Chem. 2002;277(41):38635-38646.

24. Liu Y, et al. AGEs increased migration and inflammatory responses of adventitial fibroblasts via RAGE, MAPK and NF- $\kappa B$ pathways. Atherosclerosis. 2010;208(1):34-42.

25. Zeng S, et al. Blockade of receptor for advanced glycation end product (RAGE) attenuates ischemia and reperfusion injury to the liver in mice. Hepatology. 2004;39(2):422-432.

26. Muhammad S, et al. The HMGB1 receptor RAGE mediates ischemic brain damage. J Neurosci. 2008;28(46):12023-12031.

27. Di Filippo C, Cuzzocrea S, Rossi F, Marfella R, D’Amico M. Oxidative stress as the leading cause of acute myocardial infarction in diabetics. Cardiovasc Drug Rev. 2006;24(2):77-87.

28. Sixt S, et al. Long- but not short-term multifactorial intervention with focus on exercise training improves coronary endothelia dysfunction in diabetes mellitus type 2 and coronary artery disease. Eur Heart J. 2010;31(1):112-119.

29. Yan SF, et al. RAGE and its ligands: a lasting memory in diabetic complications? Diab Vasc Dis Res. 2004;1(1):10-20.

30. Schalkwijk CG, Baidoshvili A, Stehouwer CD, van Hinsbergh VW, Niessen HW. Increased accumulation of the glycoxidation product Nepsilon-(carboxymethyl)lysine in hearts of diabetic patients: generation and characterisation of a monoclonal antiCML antibody. Biochim Biophys Acta. 2004;1636(2-3):82-89.

31. Buckingham RE, Hanna A. Thiazolidinedione insulin sensitizers and the heart: a tale of two organs? Diabetes Obes Metab. 2008;10(4):312-328.

32. Ishihara $\mathrm{M}$, et al. Comparison of blood glucose values on admission for acute myocardial infarction in patients with versus without diabetes mellitus. Am J Cardiol. 2009;104(6):769-774.

33. Vlasselaers D, et al. Tight glycemic control protects the myocardium and reduces inflammation in neonatal heart surgery. Ann Thorac Surg. 2010;90(1):22-29.

34. Nakashima A, et al. Effect of circulating soluble receptor for advanced glycation end products (sRAGE) and the proinflammatory RAGE ligand (EN-RAGE, S100A12) on mortality in hemodialysis patients. Clin J Am Soc Nephrol. 2010;5(12):2213-2219.

35. Ross R. Atherosclerosis — an inflammatory disease. N Engl J Med. 1999;340(2):115-126.

36. Aleshin A, et al. RAGE modulates myocardial injury consequent to LAD infarction via impact on JNK and STAT signaling in a murine model. Am J Physiol Heart Circ Physiol. 2008;294(4):H1823-H1832.

37. Prasanna X, Chattopadhyay A, Sengupta D. Role of lipid-mediated effects in $\beta 2$-adrenergic receptor dimerization. Adv Exp Med Biol. 2015;842:247-261.

38. Zhu WZ, et al. Heterodimerization of $\beta 1$ - and $\beta 2$-adrenergic receptor subtypes optimizes beta-adrenergic modulation of cardiac contractility. Circ Res. 2005;97(3):244-251.

39. Usami M, et al. Clinical impact of acute hyperglycemia on development of diabetes mellitus in non-diabetic patients with acute myocardial infarction. J Cardiol. 2014;63(4):274-280.

40. Hartog JW, Voors AA, Bakker SJ, Smit AJ, van Veldhuisen DJ. Advanced glycation end-products (AGEs) and heart failure: pathophysiology and clinical implications. Eur J Heart Fail. 2007;9(12):1146-1155.

41. Koyama Y, et al. High serum level of pentosidine, an advanced glycation end product (AGE), is a risk factor of patients with heart failure. J Card Fail. 2007;13(3):199-206.

42. van Heerebeek L, et al. Diastolic stiffness of the failing diabetic heart: importance of fibrosis, advanced glycation end products, and myocyte resting tension. Circulation. 2008;117(1):43-51.

43. Hartog JW, et al. Clinical and prognostic value of advanced glycation end-products in chronic heart failure. Eur Heart J. 2007;28(23):2879-2885.

44. Volz HC, et al. HMGB1: the missing link between diabetes mellitus and heart failure. Basic Res Cardiol. 2010;105(6):805-820.

45. Peters RW, et al. Mortality in the beta blocker heart attack trial: circumstances surrounding death. J Chronic Dis. 1987;40(1):75-82.

46. Hawkins CM, Richardson DW, Vokonas PS. Effect of propranolol in reducing mortality in older myocardial infarction patients Circulation. 1983;67(6 pt 2):I94-I97.

47. Sawicki PT, Siebenhofer A. Beta-blocker treatment in diabetes mellitus. J Intern Med. 2001;250(1):11-17.

48. Wai B, Kearney LG, Hare DL, Ord M, Burrell LM, Srivastava PM. Beta blocker use in subjects with type 2 diabetes mellitus and systolic heart failure does not worsen glycaemic control. Cardiovasc Diabetol. 2012;11:14.

49. Ohtsuka T, et al. Effect of beta-blockers on circulating levels of inflammatory and anti-inflammatory cytokines in patients with dilated cardiomyopathy. J Am Coll Cardiol. 2001;37(2):412-417.

50. Santilli F, Vazzana N, Bucciarelli LG, Davi G. Soluble forms of RAGE in human diseases: clinical and therapeutical implications. Curr Med Chem. 2009;16(8):940-952.

51. Lee EJ, Park JH. Receptor for Advanced Glycation Endproducts (RAGE), its ligands, and aoluble RAGE: potential biomarkers for diagnosis and therapeutic targets for human renal diseases. Genomics Inform. 2013;11(4):224-229.

52. Zhang W, Qi F, Chen DQ, Xiao WY, Wang J, Zhu WZ. Ca2+/calmodulin-dependent protein kinase II $\delta$ orchestrates G-protein-coupled receptor and electric field stimulation-induced cardiomyocyte hypertrophy. Clin Exp Pharmacol Physiol. 2010;37(8):795-802.

53. Liao RJ, et al. Rescue of cardiac failing and remodelling by inhibition of protein phosphatase 1gamma is associated with suppression of the alternative splicing factor-mediated splicing of $\mathrm{Ca}^{2+} /$ calmodulin-dependent protein kinase $\delta$. Clin Exp Pharmacol Physiol. 2014;41(12):976-985.

54. Singh MV, et al. $\mathrm{Ca}^{2+} /$ calmodulin-dependent kinase II triggers cell membrane injury by inducing complement factor B gene expression in the mouse heart. J Clin Invest. 2009;119(4):986-996.

55. Erickson JR, et al. Diabetic hyperglycaemia activates CaMKII and arrhythmias by O-linked glycosylation. Nature 2013;502(7471):372-376. 
56. Luo M, et al. Diabetes increases mortality after myocardial infarction by oxidizing CaMKII. J Clin Invest. 2013; 123(3):1262-1274.

57. Tae HJ, et al. The N-glycoform of sRAGE is the key determinant for its therapeutic efficacy to attenuate injury-elicited arterial inflammation and neointimal growth. J Mol Med (Berl). 2013;91(12):1369-1381.

58. Zhou YY, et al. Culture and adenoviral infection of adult mouse cardiac myocytes: methods for cellular genetic physiology. Am J Physiol Heart Circ Physiol. 2000;279(1):H429-H436. 